\title{
Effets des stages à l'étranger sur la formation linguistique
}

Françoise Haramboure

\section{(2) OpenEdition}

Journals

Édition électronique

URL : http://journals.openedition.org/asp/4376

DOI : 10.4000/asp.4376

ISSN : 2108-6354

Éditeur

Groupe d'étude et de recherche en anglais de spécialité

Édition imprimée

Date de publication : 1 mars 1993

Pagination : 279-286

ISSN : 1246-8185

Référence électronique

Françoise Haramboure, "Effets des stages à l'étranger sur la formation linguistique », ASp [En ligne], 1 | 1993, mis en ligne le 30 avril 2014, consulté le 19 avril 2019. URL : http://journals.openedition.org/ asp/4376 ; DOl : 10.4000/asp.4376

Ce document a été généré automatiquement le 19 avril 2019

Tous droits réservés 


\title{
Effets des stages à l'étranger sur la formation linguistique
}

\author{
Françoise Haramboure
}

1 Dans le cadre de la formation initiale, les étudiants des divers départements secondaires des I.U.T. doivent effectuer un stage de deux mois dans l'industrie en France ou à l'étranger au terme de leur cursus. L'objectif de cette expérience industrielle est triple, d'une part elle leur permet de développer leur connaissance de l'environnement industriel, d'autre part elle met en cause leur savoir - faire dans la mesure où les étudiants doivent réaliser un projet technique correspondant à un besoin de l'entreprise d'accueil, enfin elle engage leur compétence communicative dès lors que cette réalisation suppose l'intégration du stagiaire dans une équipe et la présentation orale et écrite de cette expérience.

Depuis une dizaine d'années le nombre d'étudiants effectuant leur stage en GrandeBretagne, dans un autre pays de langue anglaise ou encore dans un pays où la communication repose sur la pratique de la langue anglaise n'a cessé de croître pour atteindre vingt à vingt-cinq pour cent de chaque promotion, soit une trentaine d'étudiants chaque année.

\section{La démarche des formateurs en langue}

Conscient des limites de la formation linguistique dans le cadre institutionnel, notamment pour ce qui est du volume horaire qui lui est réservé, et de son poids relativement faible dans l'évaluation finale, le stage à l'étranger nous est apparu comme un moment et un moyen privilégiés de placer les étudiants en contact avec la langue anglaise et la culture du pays concerné. Nous nous sommes donc appuyés sur l'évolution générale des comportements initialisée par la mise en place progressive des structures européennes pour encourager les étudiants à faire leur stage dans un pays de langue anglaise. 


\section{expérience par rapport aux objectifs fixés et d'analyser ses effets sur l'apprentissage} linguistique.

Dans un premier temps, nous nous sommes fondés sur l'évaluation des maîtres de stage étrangers qui encadrent les étudiants et les commentaires spontanés des stagiaires auprès des enseignants qui leur rendent visite au cours du stage et/ou à leur retour. Ces deux sources d'informations nous ont permis d'observer que lorsque les étudiants rencontraient des difficultés, d'une part elles n'étaient pas d'ordre technique, d'autre part elles ne relevaient pas exclusivement de leurs compétences linguistiques. Pour vérifier si nos premières hypothèses étaient correctes, affiner notre analyse et conduire les étudiants à évaluer les diverses dimensions de cette expérience, nous leur avons demandé de répondre à un questionnaire individuel anonyme ou non à la fin de leur séjour à l'étranger. Cette enquête comportait quatre volets, un volet technique, un volet linguistique et culturel, un volet évaluation et un volet perspectives d'avenir.

\section{Résultats de l'évaluation}

Dans le domaine technique, la réalisation du projet de stage suppose l'acquisition de connaissances techniques nouvelles pour $80 \%$ d'entre eux. Elle constitue à ce titre un enrichissement indéniable. Cette acquisition tout comme la réalisation du projet technique dans un environnement de travail étranger, les interactions sur lesquelles elle repose avec leurs collègues de travail, la consultation de documentations, la rédaction du rapport de stage en langue étrangère, ne posent pas de problème à $90 \%$ d'entre eux.

7 En revanche, la compréhension des locuteurs natifs en dehors du cadre professionnel constitue au départ une difficulté pour $80 \%$ des stagiaires tandis que l'expression en langue étrangère est plus aisée.

8 Interrogés sur leurs suggestions pour mieux les préparer à ce type d'expérience dans le cadre de la formation initiale, $60 \%$ des étudiants proposent l'étude de documents télévisuels d'actualité "reportage TV sur la vie dans la C.E.E.", $50 \%$ d'entre eux soulignent l'importance des « débats sur l'actualité » dans les pays étrangers concernés. L'analyse des réponses fait donc émerger une demande de connaissances socio-culturelles plus approfondies pour satisfaire les exigences de la situation d'échange ou d'études dans l'environnement étranger. Si l'on corrèle ces résultats avec les observations des étudiants à l'égard de la culture étrangère, il apparaît que les aspects qui ont suscité leur étonnement couvrent un éventail très large de phénomènes allant des habitudes alimentaires aux relations hiérarchiques, au fonctionnement du système éducatif ou encore au caractère multiracial de la société britannique. De plus, si l'on procède à une analyse plus fine des questionnaires remplis par les étudiants qui ont connu des difficultés d'adaptation (10\%), les problèmes rencontrés semblent avant tout liés à leur confrontation avec une réalité différente, réticences à l'égard du mode de vie et remise en cause des habitudes sociales par exemple.

9 Les commentaires des maîtres de stage de ces derniers confirment l'hypothèse que les difficultés rencontrées sont dues dans ce cas, davantage à un rejet de la culture étrangère qu'à des obstacles purement linguistiques.

10 Dans la rubrique évaluation globale, $90 \%$ d'entre eux considèrent qu'il s'agit d'une expérience positive sur les plans techniques, linguistiques, culturels et du vécu personnel. 
Interrogés sur l'évolution de leurs compétences linguistiques au cours du séjour en fonction de leur niveau à l'arrivée dans le pays étranger, ils jugent tous avoir fait des progrès allant de 20 à $40 \%$ (pour ceux dont le niveau de départ était faible).

11 Le caractère bénéfique de l'expérience est corroboré si l'on analyse leur intention de la renouveler à l'avenir, 70 \% d'entre eux se déclarent prêts à effectuer d'autres séjours dans le même pays, $90 \%$ dans un autre pays de la C.E.E. et $80 \%$ envisagent de chercher un emploi dans un autre pays européen. Enfin, $90 \%$ d'entre eux souhaitent prolonger leur apprentissage linguistique à leur retour en France en ayant recours aux émissions de télévision en anglais/américain, à la lecture de journaux, la correspondance et les rencontres avec des locuteurs natifs, soulignant ainsi leur volonté de ne pas perdre les compétences acquises au cours du séjour.

\section{Intégration de ces données dans la formation initiale}

12 Afin de mieux préparer les étudiants à cette expérience et d'anticiper des difficultés d'adaptation éventuelles, nous avons mis en œuvre dès la première année de la scolarité une formation linguistique s'articulant simultanément sur le développement des compétences de compréhension et d'expression et une approche heuristique de la réalité socio-culturelle anglaise, américaine et européenne.

Pour favoriser cette sensibilisation aux cultures anglaise, européennes et américaine, trois types de supports d'apprentissage nous ont paru particulièrement pertinents :

- les extraits des bulletins d'information télévisés quotidiens diffusés par Continentales, CBS ou CNN, sélectionnés en fonction de leur dimension socio-culturelle;

- les programmes d'informations plus approfondis tels que Panorama portant par exemple sur un aspect particulier de la société du pays d'accueil, par exemple le caractère multi-racial de la société anglaise ou à la réunification allemande ;

- les articles de presse extraits des journaux ou revues choisis dans la même optique.

Au travail de compréhension classique permettant d'identifier les différents paramètres de l'événement présenté suivi d'une discussion ayant pour but d'élucider les causes et les conséquences de cet événement, nous avons associé un troisième volet susceptible de faire émerger des divergences et/ou des convergences avec la réalité française et au-delà un intérêt pour la culture étrangère. À cet égard, il faut souligner l'apport des documents vidéo dès lors que l'image permet souvent aux étudiants de percevoir plus concrètement certains traits spécifiques de la réalité et que l'étude du message sonore les sensibilise à l'existence de différents accents, l'association image-son mettant en évidence les relations entre les accents et le contexte géographique ou social dans lesquels ils sont susceptibles d'être entendus.

En deuxième année, l'intégration de la préparation au stage dans la formation linguistique se fait d'autant plus naturellement que l'expérience industrielle marque la fin de cette année et que les enseignants en langue sont responsables de leur organisation tout comme des poursuites d'études à l'étranger.

Dans ce cadre, la rédaction de lettres de candidature, de réponses à des propositions de stages, de curriculum vitae en langue étrangère contribuent au développement des compétences linguistiques et mettent en jeu une interaction authentique entre les étudiants et leurs interlocuteurs étrangers. 
17 L'étude du contexte dans lesquels se dérouleront les stages à partir des documents télévisés ou écrits par exemple l'article publié en janvier 1992 dans Newsweek sur Silicon Glen, région écossaise pilote dans le domaine de la haute technologie, qui n'est pas sans rappeler Silicon Valley aux États-Unis, permet aux étudiants d'appréhender des réalités économiques et industrielles susceptibles de faciliter leur adaptation à l'étranger.

18 L'analyse d'offres d'emplois parus dans les journaux britanniques destinés à des personnels de niveau équivalent est le point de départ d'une étude comparée des deux systèmes éducatifs et des conditions d'emploi dans les deux pays.

19 C'est également à une demande des étudiants que nous répondons lorsque nous introduisons des supports écrits authentiques tels que les brochures diffusées par les établissements britanniques en direction de leurs étudiants potentiels, étrangers ou non, afin de les familiariser avec l'organisation et le contenu des cursus proposés.

20 Cette sensibilisation à la réalité étrangère est approfondie par divers types de contacts. Les rencontres avec les étudiants étrangers en stage dans les entreprises de la région permettent aux apprenants de se rendre compte, grâce à l'effet miroir, du regard porté par ces derniers sur notre propre culture.

21 Les entretiens de sélection en langue étrangère avec les responsables britanniques des institutions d'accueil représentent un enjeu double pour nos étudiants, d'une part ils engagent leur avenir et, d'autre part, ils constituent une évaluation en temps réel de leurs compétences linguistiques. C'est en effet de ces dernières que dépendra leur aptitude à exposer leurs connaissances scientifiques et techniques et leurs motivations, critères déterminants dans le succès de l'entretien.

Enfin, les rencontres avec des étudiants français suivant une formation à l'étranger à leur retour en France contribuent également à cette préparation en amont. Elles sont l'occasion d'échanges d'informations indispensables à l'adaptation rapide des étudiants dans l'Université d'accueil. Elles portent notamment sur l'organisation des études, les styles d'enseignement, les relations personnelles entre étudiants et entre étudiants et enseignants.

\section{Les effets induits par cette dynamique}

23 Sur le plan personnel, il en résulte une responsabilisation des étudiants à l'égard de leur expérience à l'étranger dans la mesure où ils sont directement engagés dans sa mise en œuvre.

D'autre part l'analyse des divers aspects de la société étrangère suppose des comparaisons avec leur propre société qui les conduit à une remise en cause de leurs attitudes et pratiques et à une réflexion sur leur propre identité culturelle et sociale.

Sur le plan de l'apprentissage linguistique, outre la motivation accrue suscitée par la perspective du séjour à l'étranger, les problèmes de compréhension et/ou d'expression rencontrés au cours de la préparation induisent un investissement plus grand dans la formation linguistique, une démarche d'auto-évaluation et la recherche de solutions à court terme.

26 Ces solutions peuvent recouvrir plusieurs formes telles que le recours à l'apprentissage en autonomie avec le support des médias et l'utilisation du laboratoire de langue, et l'élaboration de stratégies individuelles d'apprentissage adaptées à leurs difficultés. 
27 L'intégration plus marquée de la dimension socio-culturelle dans la formation linguistique constitue une des réponses possibles, aux besoins des étudiants prêts à s'exposer à l'expérience de la mobilité et aux exigences de l'internationalisation des études et des emplois. Elle participe au développement d'une attitude d'empathie culturelle susceptible de favoriser la compréhension des autres et de dépasser les résistances à l'égard de la culture étrangère. Elle facilite une prise de conscience préalable des problèmes et possibilités inhérents à un séjour à l'étranger et leur permet par la suite d'en tirer un plus grand bénéfice. Par ailleurs les effets induits par la prise en compte de cette expérience dans la formation linguistique ne peuvent être ignorés par les formateurs en langue dès lors qu'ils génèrent une dynamique nouvelle dans l'apprentissage à l'intérieur des groupes d'apprenants.

\section{AUTEUR}

\section{FRANÇOISE HARAMBOURE}

Françoise Haramboure enseigne au Département GEII de IUT A Bordeaux 1.

f.Haramboure@cegetel.net 